\title{
Effects of Irisin Compared with Exercise on Specific Metabolic and Obesity Parameters in Female Mice with Obesity
}

\author{
Bahar Yuksel Ozgor, MD, Irem Demiral, MD, ${ }^{1}$ Umit Zeybek, PhD, ${ }^{2}$ Faruk Celik, PhD, ${ }^{2}$ \\ Faruk Buyru, MD, ${ }^{3}$ John Yeh, MD, ${ }^{4}$ and Ercan Bastu, $\mathrm{MD}^{3}$
}

\begin{abstract}
Objective: We aimed to analyze the changes in metabolic parameters after administration of irisin to obese female mice.

Materials and Methods: Sixty mice aged 5-6 weeks were randomized into three groups as irisin, exercise, and control. The control and irisin group remained sedentary, whereas the exercise group started free wheel exercising 6 weeks after the start of the study. The irisin group received irisin after 20 weeks. All mice were sacrificed at the 22nd week of the study, and obesity-related metabolic parameters were analyzed.

Results: There was no significant difference between the irisin and exercise groups in weight gain $(P>0.05)$. By contrast, weight gain in the control group was significantly higher compared with the irisin and exercise groups $(P<0.05)$. Serum bone morphogenetic protein $(B M P)$, ghrelin, insulin, kisspeptin, leptin, and visfatin levels were statistically lower in the irisin and exercise groups compared with the control group, but no significance was detected between the irisin and exercise groups $(P<0.05$ for all parameters).

Conclusion: Similar to the effect of exercise, irisin injections resulted in the amelioration of certain obesityrelated parameters such as the concentration of adipokines, BMP4, insulin, and ghrelin. Its role as a potential alternative to exercise needs to be further studied.
\end{abstract}

Keywords: irisin, obesity, adipokines, BMP4, ghrelin

\section{Introduction}

$\mathrm{O}$ BESITY, OFTEN REFERRED to as "the plague of the new age," is becoming an increasingly prevalent global issue. According to a recent analysis, incidences of obesity are twice as common now as they were 20 years ago. ${ }^{1}$ Individuals with obesity face numerous co-morbidities, among them diabetes, hypertension, cardiac diseases, dyslipidemia, mood disorders, and even some cancers. Immobility is one of the most significant consequences of the disease, resulting in metabolic changes and modifications to adipose tissue.

Adipose tissue does not function exclusively as a protective and insulative layer. It is also an endocrine organ and secretes a variety of hormones or peptides, known as adipocytokines, into the regulatory system; leptin, resistin, adiponectin, visfatin, and kisspeptin are among the most prominent adipocytokines produced by adipose tissue., ${ }^{2,3}$ Adipose tissue consists of important molecules that help organize the structure of fat tissue, such as a gastrointestinal system peptide, ghrelin, bone morphogenetic proteins (BMPs), and irisin (an exercise-induced myokine). BMPs are wellknown members of the transforming growth factor- $\beta$ superfamily. In the case of obesity, BMP2, BMP4, and BMP7 are well-studied molecules, and are understood to aid in the transformation of adipose tissue. ${ }^{4}$ The BMP family is known to stimulate fat tissue alteration. Studies have shown that exogenous administration of BMP4 and BMP7 cause the transformation of fat tissue into a more active variant. ${ }^{5}$

Irisin is a muscle-secreted peptide known as fibronectin type III domain-containing protein 5 (FNDC5). The secretion

\footnotetext{
${ }^{1}$ Division of Reproductive Endocrinology and Infertility, Department of Obstetrics and Gynecology, Istanbul University School of Medicine, Istanbul, Turkey.

${ }^{2}$ Institute of Experimental Medicine, Istanbul University, Istanbul, Turkey.

${ }^{3}$ Acibadem University Medical Faculty, Istanbul, Turkey.

${ }^{4}$ Department of Obstetrics and Gynecology, Massachusetts General Hospital, Harvard Medical School, Boston, Massachusetts.
} 


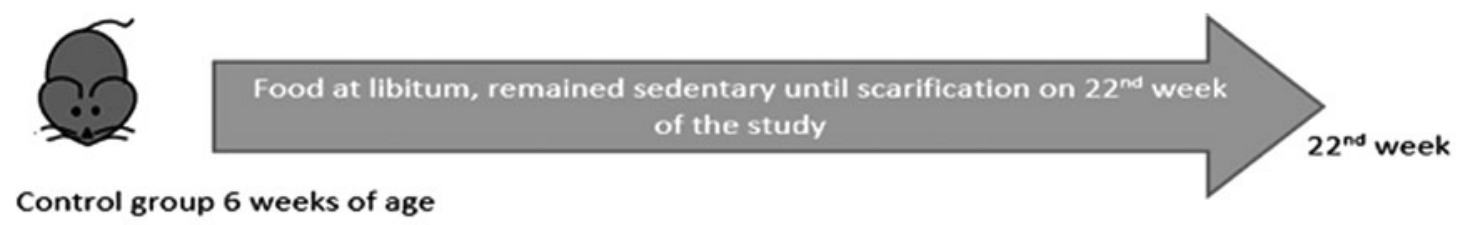

Control group 6 weeks of age
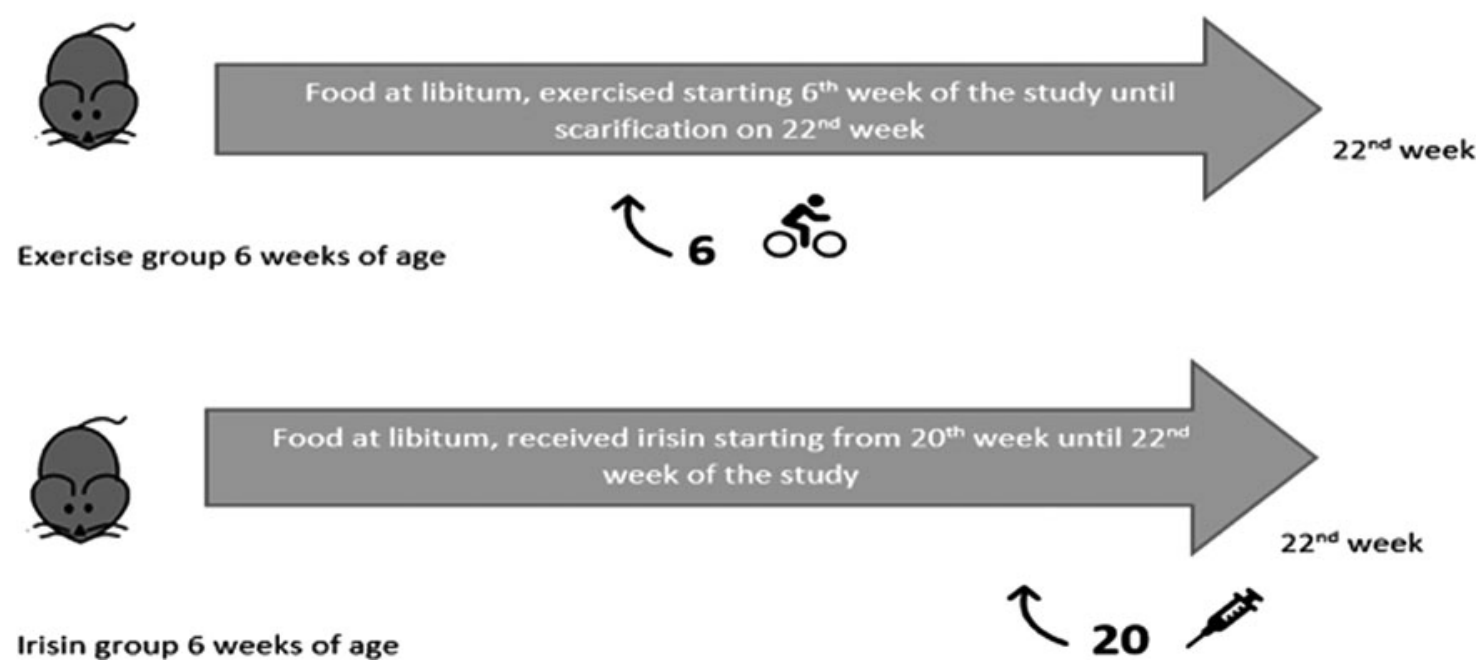

FIG. 1. Experimental model.

mechanism of irisin occurs through cleavage from FNDC5 by the induction of peroxisome proliferator-activated receptor gamma coactivator 1-alpha as a response to physical exertion or any activity that activates myokines. ${ }^{6,7}$ It is hypothesized that irisin induces transformation of fat tissue, meaning it may mimic the effects of exercise. ${ }^{8}$ Several studies show that diet-induced obesity and its related co-morbidities such as endothelial dysfunction can be reversed with irisin overexpression, thus drawing attention to irisin as a possible target for obesity treatments. ${ }^{9}$

The purpose of this investigation was to compare adipokines, BMP4 levels, and weight changes after intravenous administration of irisin and physical exercise on sedentary obese mouse populations.

\section{Material and Methods}

\section{Experimental design for mouse population}

This study is a complementary analysis of an experimentally designed female mouse population with high-fat diet-induced obesity and the effect of irisin compared with exercise. $^{10}$
The experiment was conducted in the Institute of Experimental Medicine, Istanbul University (Istanbul, Turkey), using 60 female C57BL/6J mice aged 5-6 weeks. Five mice were designated to each cage, maintained on a 14-hr light/ 10-hr dark cycle, and allowed access to food and water ad libitum for the duration of the experiment. The mice were fed a high-fat diet $(60 \% \mathrm{kcal}$, cat. no. 58Y1; Test Diet, St. Louis, MI) and maintained on the same diet until they were euthanized. Protocols of the Turkish Legislation for the Use and Care of Laboratory Animals were adhered to and ethics approval was received from the Experimental Animal Use Ethics Committee of Istanbul University.

The mouse population was divided into three groups: the irisin group $(n=20)$, exercise group $(n=20)$, and control group $(n=20)$, and were randomized as shown in Fig. 1. Mice in the irisin group remained sedentary and were injected with $10^{10}$ FNDC5-expressing adenovirus (cat. no. 041429A; Applied Biological Materials Inc., Richmond, British Columbia, Canada) beginning at the 20th week of the study until they were euthanized. The age of the mice, dosing of adenovirus, and timing were selected according to our previous study and as described by Bostrom et al. ${ }^{6,10} \mathrm{At}$

Table 1. Characteristics of Female C57Bl/6J Mice with High-Fat Diet-Induced Obesity

\begin{tabular}{|c|c|c|c|c|}
\hline & Irisin & Exercise & Control & $\mathrm{P}$ \\
\hline Weight change (grams) & $9.47 \pm 0.89^{\mathrm{a}}$ & $7.75 \pm 0.86^{\mathrm{b}}$ & $11.66 \pm 1.19^{\mathrm{a}, \mathrm{b}}$ & $<0.05$ \\
\hline HOMA-IR & $7.54 \pm 0.77^{\mathrm{a}}$ & $6.65 \pm 0.67^{\mathrm{b}}$ & $9.31 \pm 1.23^{\mathrm{a}, \mathrm{b}}$ & $<0.05$ \\
\hline Irisin (ng/mL) & $742.02 \pm 282.07^{\mathrm{a}}$ & $830.96 \pm 303.56^{\mathrm{b}}$ & $631.95 \pm 201.99^{\mathrm{a}, \mathrm{b}}$ & $<0.05$ \\
\hline $\mathrm{E} 2(\mathrm{pg} / \mathrm{mL})$ & $2,441.50 \pm 1,062.62$ & $2,568.58 \pm 1,174.19$ & $3,080.54 \pm 1,887.93$ & NS \\
\hline $\mathrm{LH}(\mathrm{mIU} / \mathrm{mL})$ & $85.00 \pm 32.21^{\mathrm{a}}$ & $70.95 \pm 9.46$ & $59.78 \pm 32.73^{\mathrm{a}}$ & $<0.05$ \\
\hline $\mathrm{FSH}(\mathrm{ng} / \mathrm{mL})$ & $117.48 \pm 39.78$ & $111.50 \pm 17.74$ & $108.72 \pm 28.63$ & NS \\
\hline
\end{tabular}

Similar superscript letters indicate a statistically significant difference $(P<0.05)$.

E2, estradiol; FSH, follicle-stimulating hormone; HOMA-IR, homeostatic model assessment of insulin resistance; LH, luteinizing hormone; NS, not significant at $P>0.05$. 
Table 2. Comparison of Metabolic Parameters Between Irisin, Exercise, and Control Groups

\begin{tabular}{|c|c|c|c|c|}
\hline & Irisin & Exercise & Control & $\mathrm{P}$ \\
\hline Adiponectin (mg/L) & $105.7 \pm 34.5$ & $90.4 \pm 51.7$ & $76.6 \pm 43.7$ & NS \\
\hline ANP (ng/L) & $979.3 \pm 267.6$ & $1,136.5 \pm 600.5$ & $847.7 \pm 348.5$ & NS \\
\hline $\mathrm{BMP}(\mathrm{ng} / \mathrm{L})$ & $2,927.5 \pm 712.4^{\mathrm{a}}$ & $3,034.1 \pm 1,144.4^{\mathrm{b}}$ & $12,210 \pm 740.9^{\mathrm{a}, \mathrm{b}}$ & $<0.05$ \\
\hline BNP (ng/L) & $549.0 \pm 223.9$ & $680.7 \pm 374.0$ & $541.1 \pm 48.9$ & NS \\
\hline FGF21 (pg/mL) & $1,176.6 \pm 376.8^{\mathrm{a}}$ & $1,473.6 \pm 819.6$ & $1,633 \pm 551.4^{\mathrm{a}}$ & $<0.05$ \\
\hline Ghrelin (pg/mL) & $5,314.3 \pm 2,710.9^{a}$ & $5,906.0 \pm 1,504.9^{b}$ & $15,055.9 \pm 2,762.2^{\mathrm{a}, \mathrm{b}}$ & $<0.05$ \\
\hline Insulin (mIU/L) & $49.5 \pm 19.7^{\mathrm{a}}$ & $44.2 \pm 17.8^{\mathrm{b}}$ & $138 \pm 16.9^{\mathrm{a}, \mathrm{b}}$ & $<0.05$ \\
\hline Kisspeptin $(\mathrm{pg} / \mathrm{mL})$ & $2,509.2 \pm 895.9^{\mathrm{a}}$ & $2,117.6 \pm 642.7^{\mathrm{b}}$ & $3,340.4 \pm 313.8^{\mathrm{a}, \mathrm{b}}$ & $<0.05$ \\
\hline Leptin (ng/L) & $2,789.2 \pm 1,362.1^{\mathrm{a}}$ & $2,572.5 \pm 1,087.5^{\mathrm{b}}$ & $5,599.9 \pm 321.0^{\mathrm{a}, \mathrm{b}}$ & $<0.05$ \\
\hline RBP4 (ng/mL) & $398.8 \pm 132.8$ & $339.8 \pm 87.3$ & $432.2 \pm 221.5$ & NS \\
\hline Resistin (ng/mL) & $131.0 \pm 65.2$ & $115.7 \pm 39.5^{\mathrm{b}}$ & $158.8 \pm 22.7^{\mathrm{b}}$ & $<0.05$ \\
\hline Visfatin (ng/mL) & $482.1 \pm 155.4^{\mathrm{a}}$ & $443.7 \pm 175.4^{\mathrm{b}}$ & $1,388.8 \pm 226.7^{\mathrm{a}, \mathrm{b}}$ & $<0.05$ \\
\hline
\end{tabular}

Similar superscript letters indicate a statistically significant difference $(P<0.05)$

ANP, atrial natriuretic factor; BMP, bone morphogenetic protein; BNP, brain natriuretic factor; FGF21, fibroblast growth factor 21; RBP4, retinol-binding protein 4 ; NS, not significant at $P>0.05$.

the 6th week of the study, at $\sim 12$ weeks of age, the mice in the exercise group began exercising using freewheel running until the end of the study, when they were euthanized at the 22nd week. The controls remained sedentary from the beginning of the experiment until they were euthanized. One of the exercise mice died of an unknown cause.

\section{Physical activity assessment}

Free wheel running systems were installed and all mice in the exercise group used wheels for 6 weeks from the beginning of the study (Fig. 1).

\section{Definition of obesity}

The mice were weighed every 2 weeks and their weights were documented. To standardize the results, the homeostatic model assessment insulin resistance (HOMA-IR) index was determined at the end of the study using the following formula: (fasting insulin [mIU/L] fasting glucose $(\mathrm{nmol} / \mathrm{L}) / 22.5$. All HOMA data are presented in Table 1.

\section{Adipokine and hormone levels}

Blood samples were collected after $12 \mathrm{hr}$ of fasting. Mouse blood was obtained through cardiac puncture immediately after euthanasia. After centrifugation, serum was separated into $15-\mu \mathrm{L}$ vials and stored at $-80^{\circ} \mathrm{C}$ until required for evaluation. At the time of testing, serum was used for enzyme-linked immunosorbent assay (ELISA) analysis. Ghrelin, insulin, kisspeptin, visfatin, adiponectin, atrial natriuretic peptide (ANP), BMP, estradiol, folliclestimulating hormone, luteinizing hormone, irisin, retinolbinding protein 4 (RBP4), and resistin were measured using commercially available ELISA kits according to the manufacturer's instructions, keeping inter- and intra-assay coefficients of variations $<10 \%$ (Merck Millipore, Darmstadt, Germany). Serum BMP4 levels were measured using a BMP-4 ELISA kit (cat. no.\# ab99982; Abcam, Cambridge, MA). Adipokines (leptin and resistin) were measured using a mouse gut hormone magnetic bead panel (Millipore, Billerica, MA) according to the manufacturer's instructions.

\section{Statistical analysis}

The mean value was used to present continuous variables (standard deviation). All variables were distributed normally in accordance with histograms. The comparison of two groups was accomplished using Student's $t$-test, and three groups were analyzed using one-way analysis of variance (ANOVA), followed by post hoc Tukey Tests. Pearson's correlation coefficient was chosen for pairwise correlations of hormonal and selected mediators. Analyses were performed using version 20.0 of the Statistical Package for the Social Sciences software (SPSS, Inc., Chicago, IL) and version 6 of GraphPad Prism software (GraphPad Software, Inc., La Jolla, CA). A $P<0.05$ was considered significant.

\section{Results}

In this investigation we found no significant difference between the irisin and exercise groups $(9.47$ vs. $7.75 \mathrm{~g}$; $P>0.05)$ with respect to weight change. The weight change in the control group was significantly greater compared with the irisin and exercise groups $(P<0.05)$. The HOMA-IR, which was used to check the risk of metabolic syndrome, showed no difference between the irisin and exercise groups $(P>0.05)$, although it was significantly higher in the control group $(P<0.05)$. The characteristics of the female mice with high-fat diet-induced obesity are listed in Table 1.

Serum adiponectin, ANP, BNP, RBP4, and resistin levels showed no significant difference between the irisin and control groups $(P>0.05)$. The serum levels of the same molecules showed a similarity in the exercise group, whereas serum resistin levels were significantly reduced in the exercise group compared with the control group (115.7 vs. $158.8 \mathrm{ng} / \mathrm{mL}$, $P<0.05)$. In contrast, resistin levels were not significantly different between the irisin and exercise groups (131.0 and $115.7 \mathrm{ng} / \mathrm{mL}$, respectively; $P>0.05$ ). FGF21 levels were lower in both the irisin and exercise groups compared with the controls, showing a statistical difference in the irisin group $(P<0.05)$. Serum BMP, ghrelin, insulin, kisspeptin, leptin, and visfatin concentrations were statistically lower in the irisin group compared with the control group (2,927.5 vs. $12,210 \mathrm{ng} / \mathrm{L}, 5,314.3$ vs. $15,055.9 \mathrm{pg} / \mathrm{mL}, 49.5$ vs. $138 \mathrm{mIU} / \mathrm{L}$, $2,509.2$ vs. $3,340.4 \mathrm{pg} / \mathrm{mL}, 2,789.2$ vs. $5,599.9 \mathrm{ng} / \mathrm{L}, 482.1$ vs. 
$1,388.8 \mathrm{ng} / \mathrm{mL}, P<0.05$ for all parameters, respectively). Similarly, serum BMP, ghrelin, insulin, kisspeptin, leptin, and visfatin concentrations were significantly lower in the exercise group in comparison with the controls. Moreover, there were no statistical differences between BMP, ghrelin, insulin, kisspeptin, leptin, and visfatin serum concentrations in the irisin and exercise groups (Table 2).

\section{Discussion}

In the study, irisin injection resulted in exercisemimicking effects on metabolic parameters related to obesity. The mice in the irisin group gained only a little more weight than the exercise group, even when not exercising under sedentary conditions. All metabolic parameters were positively altered in the irisin group similar to the exercise group.

Abdominal obesity, determined by fat tissue, is a risk for metabolic syndrome and is known to cause cardiovascular disease and type 2 diabetes. Today, it is believed that the underlying factors for the adverse effects of fat tissue arise from the excess release of adipokines from fat tissue cells into the blood system. ${ }^{11,12}$ The adipokines secreted from enlarged adipocytes (e.g., leptin, ghrelin, kisspeptin, and resistin), and cytokines from the newly arrived inflammatory cells, cause systemic inflammation and metabolic syndrome. ${ }^{13-15}$ These findings led to hypotheses involving lowering adipokine levels to find a solution to obesity. AbuFarha et al. reported that groups with elevated levels of more than two proinflammatory adipokines had higher rates of metabolic syndrome compared with those with less than two proinflammatory adipokines. ${ }^{16}$ According to Meiliana et al., the prevalence of obesity and metabolic syndrome changed with the levels of adipokines between the obese group and control group. ${ }^{17}$

The effects of exercise on fat tissue have been well documented, showing an increase in lipolysis and glucose exchange. Stanford et al. claimed that transplantation of fat tissue from exercising mice into sedentary mice helped the recipients improve their glucose homeostasis. ${ }^{18}$ The mechanism of the molecular changes caused by exercising is still controversial; however, one of the strongest candidates is elevated quantities of irisin molecules.

Irisin is a polypeptide cleaved from FNDC5A. Boström et al. showed an increase in serum irisin levels after 8 weeks of voluntary wheel exercising mice. ${ }^{6}$ They explained the mechanism as PGC1- $\alpha$ expression in muscle, which stimulated an increase in the expression of FNDC5. Recent studies also showed the role of irisin in myoblast proliferation and muscle development. ${ }^{19}$ These findings encouraged hypotheses of exercise-mimicking effects produced by irisin administration, as seen in the recent study by Ye et al., which defined irisin as an agent reversing insulin. ${ }^{20}$

Our results show that both exercise and irisin administration produce similar outcomes on serum BMP, ghrelin, insulin, kisspeptin, leptin, and visfatin concentrations. Recently, a human study showed that administration of exenatide, an inhibitor of BMP4, to obese patients resulted in an improvement in obesity-related diseases and changes in body fat distribution. ${ }^{21}$ Although there are no investigations that demonstrate the relation between BMP4 levels and irisin administration, our results are in correlation with BMP decrease-based outcomes from previous studies.
The predominant molecules associated with obesity are adipokines (leptin, visfatin, and kisspeptin), and a gastrointestinal system peptide, ghrelin. ${ }^{22}$ Leptin levels in the blood system usually correlate with adipocyte production, which represents the amount of adipose tissue. ${ }^{23}$ Serum leptin concentrations are higher in individuals with obesity compared with normal weight individuals. Our results showed a decrease in leptin and insulin levels both with irisin administration and exercise.

Visfatin and kisspeptin are also derived from adipose tissue. Visfatin is known to capture glucose into adipocytes and prevent its release resulting in an increase in glucose-tolipid translation. ${ }^{24}$ Until recently, it was thought that kisspeptin was only derived from the brain and had functions in reproduction, but it is now known that it has other sources and targets such as liver, adipose tissue, and pancreas, suggesting a role in metabolism and metabolic syndrome. ${ }^{25,26}$ Thus, the serum levels of kisspeptin seem to be a possible target for next-generation obesity treatments.

Ghrelin is a gastrointestinal system-secreted peptide that has a unique structure with 28 amino acids and an $n$-octanoyl ester at its third serine residue. It is a well-known orexigenic hormone that stimulates food intake. ${ }^{27}$ The effect of precipitating hunger is increased in a dose-dependent manner. Hence, the amount of ghrelin in circulation seems related to obesity caused by excess food intake. Exercise appears to break the chain by decreasing the amount of ghrelin, as irisin application does, or change the appetite.

There were several limitations on our investigation. The number of available animal subjects for use in the study was relatively small. Furthermore, the techniques we used to measure the quantity of molecules can be differentiated by using different assay modalities. ${ }^{28,29}$ It is our hypothesis that our findings might be reproducible in humans, but the data cannot be transferred directly; only through human testing can this be confirmed.

\section{Conclusion}

In conclusion, obesity is still the greatest battle of our age, and the only proven tool we have against obesity is exercise. Similar to the effect of exercise, irisin injections resulted in the amelioration of obesity-related parameters such as the concentration of adipokines, BMP4, insulin, and ghrelin. Its role as a potential alternative to exercise needs to be further studied.

\section{Acknowledgments}

The authors thank David F. Chapman, BSc, and Chanel Schroff for editing the language of the article.

\section{Author Disclosure Statement}

No conflicting financial interests exist.

\section{Funding Information}

No funding was received for this article.

\section{References}

1. Kopelman PG. Obesity as a medical problem. Nature 2000; 404:635-643.

2. Batitucci G, Brandao CFC, De Carvalho FG, et al. Taurine supplementation increases irisin levels after high intensity physical training in obese women. Cytokine 2019;123:154741. 
3. Gesta S, Tseng YH, Kahn CR. Developmental origin of fat: Tracking obesity to its source. Cell 2007;131:242-256.

4. Bowers RR, Kim JW, Otto TC, et al. Stable stem cell commitment to the adipocyte lineage by inhibition of DNA methylation: Role of the BMP-4 gene. Proc Natl Acad Sci U S A 2006;103:13022-13027.

5. Tseng YH, Kokkotou E, Schulz TJ, et al. New role of bone morphogenetic protein 7 in brown adipogenesis and energy expenditure. Nature 2008;454:1000-1004.

6. Boström P, Wu J, Jedrychowski MP, et al. A PGC1- $\alpha$ dependent myokine that drives brown-fat-like development of white fat and thermogenesis. Nature 2012;481:463.

7. Handschin C, Spiegelman BM. The role of exercise and PGC1alpha in inflammation and chronic disease. Nature 2008;454:463-469.

8. Hecksteden A, Wegmann M, Steffen A, et al. Irisin and exercise training in humans - results from a randomized controlled training trial. BMC Med 2013;11:235.

9. Hou N, Du G, Han F, et al. Irisin regulates heme oxygenase-1/adiponectin axis in perivascular adipose tissue and improves endothelial dysfunction in diet-induced obese mice. Cell Physiol Biochem 2017;42:603-614.

10. Bastu E, Zeybek U, Gurel Gurevin E, et al. Effects of irisin and exercise on metabolic parameters and reproductive hormone levels in high-fat diet-induced obese female mice. Reprod Sci 2018;25:281-291.

11. Alberti G, Zimmet PZ. Definition, diagnosis and classification of diabetes mellitus and its complications. Part 1: Diagnosis and classification of diabetes mellitus. Provisional report of a WHO Consultation. Diabet Med 1998;15: 539-553.

12. Després J-P. Abdominal obesity: The most prevalent cause of the metabolic syndrome and related cardiometabolic risk. Eur Heart J Suppl 2006;8(suppl_B):B4-B12.

13. Xu H, Barnes GT, Yang Q, et al. Chronic inflammation in fat plays a crucial role in the development of obesity-related insulin resistance. J Clin Invest 2003;112:1821-1830.

14. Lumeng CN, Bodzin JL, Saltiel AR. Obesity induces a phenotypic switch in adipose tissue macrophage polarization. J Clin Invest 2007;117:175-184.

15. Fontana L, Eagon JC, Trujillo ME, et al. Visceral fat adipokine secretion is associated with systemic inflammation in obese humans. Diabetes 2007;56:1010-1013.

16. Abu-Farha M, Behbehani K, Elkum N. Comprehensive analysis of circulating adipokines and hsCRP association with cardiovascular disease risk factors and metabolic syndrome in Arabs. Cardiovasc Diabetol 2014;13:76.

17. Meiliana A, Wijaya A, As'ad Armyn S. The relationship of proinflammatory and antiinflammatory adipokines in the development of metabolic syndrome in centrally obese men. Indonesian Biomed J 2010;2:118.
18. Stanford KI, Middelbeek RJ, Townsend KL, et al. A novel role for subcutaneous adipose tissue in exercise-induced improvements in glucose homeostasis. Diabetes 2015;64: 2002-2014.

19. Reza MM, Subramaniyam N, Sim CM, et al. Irisin is a promyogenic factor that induces skeletal muscle hypertrophy and rescues denervation-induced atrophy. Nat Commun 2017;8.1:1104.

20. Ye, $\mathrm{X}$, Shen YM, Ni C, et al. Irisin reverses insulin resistance in $\mathrm{C} 2 \mathrm{C} 12$ cells via the p38-MAPK-PGC-1 $\alpha$ pathway. Peptides 2019;119:170120.

21. Wang X, Chen J, Li L, et al. New association of bone morphogenetic protein 4 concentrations with fat distribution in obesity and exenatide intervention on it. Lipids Health Dis 2017;16:70.

22. Deng Y, Scherer PE. Adipokines as novel biomarkers and regulators of the metabolic syndrome. Ann N Y Acad Sci 2010;1212:E1-E19.

23. Maffei M, Halaas J, Ravussin E, et al. Leptin levels in human and rodent: Measurement of plasma leptin and ob RNA in obese and weight-reduced subjects. Nat Med 1995; 1:1155-1161.

24. Skop V, Kontrová K, Zídek V, et al. Autocrine effects of visfatin on hepatocyte sensitivity to insulin action. Physiol Res 2010;59:615-618.

25. Lapatto R, Pallais JC, Zhang D, et al. Kiss1-/- mice exhibit more variable hypogonadism than Gpr54-/- mice. Endocrinology 2007; 148:4927-4936.

26. Tolson KP, Garcia C, Yen S, et al. Impaired kisspeptin signaling decreases metabolism and promotes glucose intolerance and obesity. J Clin Invest 2014;124:3075-3079.

27. Cheung CK, Wu JC. Role of ghrelin in the pathophysiology of gastrointestinal disease. Gut Liver 2013;7:505-512.

28. Wang K, Wadhwa PD, Culhane JF, et al. Validation and comparison of luminex multiplex cytokine analysis kits with ELISA: Determinations of a panel of nine cytokines in clinical sample culture supernatants. J Reprod Immunol 2005;66:175-191.

29. Gomez-Banoy N, Guseh JS, Li G, et al. Adipsin preserves beta cells in diabetic mice and associates with protection from type 2 diabetes in humans. Nat Med 2019;25:1739-1747.

Address correspondence to: Bahar Yuksel Ozgor, MD

Division of Reproductive Endocrinology and Infertility Department of Obstetrics and Gynecology Istanbul University School of Medicine

Istanbul 34100

Turkey

E-mail: bahary186@gmail.com 